\title{
Systematic Extended Right Posterior Sectionectomy (SERPS) for secondary liver tumors, a tale of two cases
}

\author{
Michael TENDEAN*, Toar.D.B. MAMBU, Ferdinand TJANDRA
}

Surgery Department, Digestive Surgery Division, Prof. dr. R.D. Kandou General Hospital, Indonesia

Introduction: The role of liver resection in secondary liver tumors are growing along with the consequences of Post Hepatectomy Liver Failure (PHLF). Systematic Extended Right Posterior Sectionectomy (SERPS) is offered as an alternative to resect secondary liver tumors in segment 6-7 with vascular invasion towards right hepatic vein (RHV) compared to the right hepatectomy procedure in the past. The goal of SERPS procedure itself is maintaining appropriate oncological border, while minimizing the risk of PHLF.

Methods: We aim to describe two patients with liver metastases in segment 6-7 with RHV vascular invasion, due to GIST and colorectal cancer metastases, which undergone USG guided SERPS in 2020. We reported the patients' primary tumor characteristics, adjuvant chemotherapy, surgical outcomes, PHLF incidence measured with ISGLS criteria, and liver metastases recurrencies in 3 months post-operative.

Results: No tumor recurrencies and mortalities due to primary tumors or complications were detected in 3 months post-operative follow-ups. No PHLF incidence were detected.

Conclusions: SERPS is a safe and promising alternative for secondary liver tumors at segment 6-7 and RHV vascular invasion. Thus, minimizing the risk of PHLF by saving larger volume of future liver remnant. 